\title{
FINDING LINEAR PATTERNS OF COMPLEXITY ONE
}

\author{
XUANCHENG SHAO
}

\begin{abstract}
We study the following generalization of Roth's theorem for 3-term arithmetic progressions. For $s \geq 2$, define a nontrivial $s$-configuration to be a set of $s(s+1) / 2$ integers consisting of $s$ distinct integers $x_{1}, \cdots, x_{s}$ as well as the averages $\left(x_{i}+x_{j}\right) / 2(1 \leq i<j \leq s)$. Our main result states that if a set $A \subset[N]$ has density $\delta \gg(\log N)^{-c(s)}$ for some positive constant $c(s)>0$ depending on $s$, then $A$ contains a nontrivial $s$-configuration. This improves on the previous bound of the form $\delta \gg(\log \log N)^{-c(s)}$ due to Dousse [3]. We also deduce, as a corollary, an improvement of a problem involving sumfree subsets.
\end{abstract}

\section{INTRODUCTION}

The celebrated Roth's theorem [11] states that every dense subset of the integers contains a nontrivial 3-term arithmetic progression (3-AP).

Theorem (Roth). Let $0<\delta<1$ be a positive real. For a sufficiently large positive integer $N$, any subset $A \subset[N]$ with $|A| \geq \delta N$ must contain a nontrivial 3 -term arithmetic progression.

Here we use $[N]$ to denote the set of positive integers up to $N$. In fact, Roth obtained the more precise bound $\delta \gg(\log \log N)^{-1}$. This has been subsequently improved by Szemerédi [17] and Heath-Brown [10] to $\delta \gg(\log N)^{-c}$ for some small positive constant $c$. Later Bourgain [1,2] improved this to $\delta \gg(\log N)^{-2 / 3+o(1)}$. Currently the best quantitative bound is due to Sanders [15]: $\delta \gg(\log N)^{-1+o(1)}$. On the other hand, a construction of Behrend shows that there exists a subset without nontrivial 3-APs with density about $\exp (-C \sqrt{\log N})$ for some constant $C>0$.

Roth's argument relies on a dichotomy which either guarantees the existence of a nontrivial 3-AP, or leads to a density increment in a shorter subprogression. See [19] for an exposition of Roth's density increment argument as well as some of the later improvements.

We are interested in the following generalization of 3-APs. Fix a positive integer $s \geq 2$. An $s$-configuration is set of $s(s+1) / 2$ integers

$$
\left\{n_{i}+n_{j}+a: 1 \leq i \leq j \leq s\right\},
$$

where $n_{1}, \cdots, n_{s}, a \in \mathbb{Z}$. For example, when $s=2$, the three integers $\left(2 n_{1}+a, n_{1}+n_{2}+\right.$ $\left.a, 2 n_{2}+a\right)$ form a 3-AP. An $s$-configuration is said to be nontrivial if $n_{1}, \cdots, n_{s}$ are all distinct. A geometrical way to think about $s$-configurations is that they contain $s$ points on the real line and all midpoints between any two of these points.

It is natural to ask whether every dense subset of the integers contains a nontrivial $s$ configuration (for fixed $s$ ). This is proved by Dousse [3] using Roth's density increment argument. 
Theorem 1.1. Let $A \subset[N]$ with $|A|=\delta N$. Suppose that

$$
\delta \gg(\log \log N)^{-\frac{1}{s(s+1)-1}} .
$$

Then A contains a nontrivial s-configuration.

A crucial reason why Roth's argument applies to $s$-configurations is that the set of linear forms in (1.1) has complexity 1 (regardless of the value of $s$ ), and thus controlled by Gowers $U^{2}$-norm. The Gowers uniformity norms are the main tools in Gowers' proof [4,5] of Szemerédi's theorem. The notion of complexity of linear forms seems to originate from [9]; for the precise definition and related results see [18].

For comparison, the set of linear forms corresponding to $(k+1)$-APs has complexity $k-1$, and thus controlled by Gowers $U^{k}$-norm. While analyzing the $U^{2}$-norm is more or less equivalent to doing Fourier analysis, dealing with the $U^{k}$-norm when $k>2$ becomes significantly more difficult. Indeed this is the main topic of the book [18]. Fortunately for us, to study $s$-configurations it is sufficient to consider the $U^{2}$-norm.

We now state our main result:

Theorem 1.2. Let $A \subset[N]$ with $|A|=\delta N$. Suppose that

$$
\delta \geq 100\left(\frac{\log \log N}{\log N}\right)^{1 / 6 s(s+1)} .
$$

Then A contains a nontrivial s-configuration.

The exponent $1 / 6 s(s+1)$ can certainly be improved slightly by a more careful analysis. We shall not do so here. Our argument should work for other linear patterns of complexity 1 (and thus the title for this paper). However doing so in this generality involves some technical complications. We will thus focus solely on $s$-configurations.

The proof of Theorem [1.2 relies on Fourier analysis localized at certain approximate subgroups called Bohr sets. This technique is originated by Bourgain [1] in his work on finding 3 -APs and developed further in [6,14]. In order for the local Fourier analysis argument to work on $s$-configurations, we need a local analogue of Gowers $U^{2}$-norm; this is the main innovation of this paper.

It is worth mentioning that a weaker bound of the form $\delta \gg \exp (-c(s) \sqrt{\log \log N})$ can be obtained by following the argument of Green-Tao [8], where the problem of finding 4APs is studied. The main idea behind their argument is in turn borrowed from Szemerédi and Heath-Brown's work in the case of 3-APs. Our problem with $s$-configurations that are governed by Gowers $U^{2}$-norm is much simpler than the problem with 4-APs that are governed by Gowers $U^{3}$-norm. It is thus not surprising that we are able to get a better bound for $s$-configurations. In the ongoing work of Green-Tao they show that a bound of the same quality can be obtained in the case of 4-APs as well.

The improved bound in Theorem 1.2, combined with the argument of Sudakov, Szemerédi, and $\mathrm{Vu}$ [16], leads to an improvement in a problem involving sumfree sets. 
Corollary 1.3. Any set $A \subset \mathbb{Z}$ of size $n$ contains a subset $B \subset A$ with size $|B| \geq$ $(\log n)(\log \log n)^{1 / 2-o(1)}$ such that $B$ is sumfree with respect to $A$. In other words, $b+b^{\prime} \notin A$ for any two distinct elements $b, b^{\prime} \in B$.

Here $o(1)$ denotes a quantity that goes to zero as $n \rightarrow \infty$. For a more detailed discussion on this problem we refer the reader to [16], where the first superlogarithmic bound on $|B|$ is obtained. The bound $|B| \geq(\log n)(\log \log \log n)^{c}$ is obtained by Dousse [3] using Theorem 1.1. In exactly the same way, Theorem 1.2 immediately gives the bound $|B| \geq$ $(\log n)(\log \log n)^{c}$ for some small positive constant $c>0$. The improvement of the exponent $c$ is a consequence of using the Ruzsa embedding lemma instead of Freiman's theorem to extract a dense subset of a long interval from a set with small doubling.

The rest of the paper is organized as follows. In Section 2 we collect basic properties of Bohr sets, a multi-dimensional analogue of arithmetic progressions. Theorem 1.2 is then proved in Sections 3 to 5 . Section 3 develops the theory of a local analogue of Gowers $U^{2}$-norm; this captures the intuition of performing Fourier analysis on Bohr sets (approximate groups) instead of genuine groups. In Section 4 we establish a dichotomy which either guarantees the existence of a nontrivial $s$-configuration or leads to a large local $U^{2}$-norm. Finally in Section 5 we obtain a density increment from the large local $U^{2}$-norm and complete the proof of Theorem 1.2. The last section contains the proof of Corollary 1.3.

Acknowledgements. The author is grateful to Ben Green and Terry Tao for some helpful discussions.

\section{Bohr Sets: Basic Properties}

In this section we record some basic properties of Bohr sets, all of which can be found in standard texts in additive combinatorics such as [19].

Definition 2.1 (Bohr sets). Let $\theta=\left(\theta_{1}, \cdots, \theta_{d}\right) \in \mathbb{R}^{d}, 0<\epsilon<1 / 2$, and $M \geq 1$. Define the Bohr set $\Lambda=\Lambda_{\theta, \epsilon, M}$ to be the set of all $n \in \mathbb{Z}$ with $|n| \leq M$ and $\left\|n \theta_{j}\right\| \leq \epsilon$ for each $1 \leq j \leq d$ (here $\|x\|$ denotes the distance from $x$ to its nearest integer). The positive integer $d$ is called the dimension of $\Lambda$. For any real number $c>0$, we write $c \Lambda$ for the dilated Bohr set $c \Lambda=\Lambda_{\theta, c \epsilon, c M}$.

For example, the interval $[-M, M]$ is the 1-dimensional Bohr set $\Lambda_{1,1 / 2, M}$. A simple heuristic argument shows that an integer $n \in[-M, M]$ lies in a $d$-dimensional Bohr set $\Lambda_{\theta, \epsilon, M}$ with probability $\epsilon^{d}$, and thus $\Lambda_{\theta, \epsilon, M}$ has expected size roughly $\epsilon^{d} M$. This is indeed correct as a lower bound.

Lemma 2.2 (Size bound). Let $\Lambda=\Lambda_{\theta, \epsilon, M}$ be a Bohr set of dimension $d$. Then $|\Lambda| \geq \epsilon^{d} M$.

Bohr sets are approximate groups in the sense that $|\Lambda+\Lambda| \approx 2^{d}|\Lambda|$. However, as $d$ gets large the doubling also gets large. To get around this unpleasant behavior, we will work with pairs of Bohr sets $(\Lambda, c \Lambda)$ for some small constant $c \in(0,1)$. Heuristically we expect that $|\Lambda+c \Lambda| \approx|\Lambda|$. This leads to the following definition. 
Definition 2.3 (Regular Bohr sets). A Bohr set $\Lambda=\Lambda_{\theta, \epsilon, M}$ of dimension $d$ is said to be regular if

$$
1-100 d|c| \leq \frac{|(1+c) \Lambda|}{|\Lambda|} \leq 1+100 d|c|
$$

whenever $|c| \leq 1 / 100 d$.

Not all Bohr sets are regular. For example, consider $\Lambda=\Lambda_{1 / 2,0.499, M}$, and observe that while $\Lambda$ contains only even integers in $[-M, M]$, the dilated Bohr set $1.01 \Lambda$ contains all integers in $[-M, M]$. On the other hand, it is not hard to see that $\Lambda_{1 / 2, \epsilon, M}$ is regular when $\epsilon$ is not too close to 0.5. In general, the following lemma shows that regular Bohr sets exist in abundance.

Lemma 2.4 (Finding regular Bohr sets). For any Bohr set $\Lambda$, there exists $\alpha \in[1 / 2,1]$ such that $\alpha \Lambda$ is regular.

Suppose that $\Lambda$ is a regular Bohr set of dimension $d$, and $\Lambda^{\prime}=c \Lambda$ for some small constant $0<c<1 / 100 d$. As mentioned above, the approximate group structure of the pair $\left(\Lambda, \Lambda^{\prime}\right)$ is crucial in our argument. For example, let $f: \mathbb{Z} \rightarrow \mathbb{C}$ be a 1-bounded function (meaning that $|f| \leq 1$ ), and consider the average

$$
\mathbb{E}_{n \in n^{\prime}+\Lambda} f(n)
$$

for some $n^{\prime} \in \Lambda^{\prime}$. The regularity of $\Lambda$ allows us to replace the range $n \in n^{\prime}+\Lambda$ by $n \in \Lambda$, at the cost of a small error:

$$
\mathbb{E}_{n \in n^{\prime}+\Lambda} f(n)=\mathbb{E}_{n \in \Lambda} f(n)+O(c d) .
$$

We will use this type of estimate again and again without explicitly mentioning it.

\section{The Local Gowers $U^{2}$-NORM}

In this section, we define a local Gowers $U^{2}$-norm and prove an inverse theorem for it. To motivate the definition, first recall the usual $U^{2}$-norm, defined for a 1-bounded function $f: G \rightarrow \mathbb{C}$ on a finite abelian group $G$ :

$$
\|f\|_{U^{2}}^{4}=\mathbb{E}_{a, d_{1}, d_{2} \in G} f(a) \overline{f\left(a+d_{1}\right) f\left(a+d_{2}\right)} f\left(a+d_{1}+d_{2}\right) .
$$

Geometrically $\|f\|_{U^{2}}$ counts parallelograms weighted by $f$, with the parameter $a$ representing one of the vertices of the parallelogram, and $d_{1}, d_{2}$ representing the two edges of it.

Definition 3.1 (Local Gowers $U^{2}$-norm). Let $\Lambda, \Lambda_{1}=c_{1} \Lambda, \Lambda_{2}=c_{2} \Lambda_{1}$ be regular Bohr sets of dimension $d$, where $0<c_{1}, c_{2}<1 / 100 d$. For a 1 -bounded function $f: \mathbb{Z} \rightarrow \mathbb{C}$, its local Gowers $U^{2}$-norm with respect to $\left(\Lambda, \Lambda_{1}, \Lambda_{2}\right)$ is defined by

$\|f\|_{U^{2}\left(\Lambda, \Lambda_{1}, \Lambda_{2}\right)}^{4}=\mathbb{E}_{a \in \Lambda} \mathbb{E}_{n_{1}, n_{1}^{\prime} \in \Lambda_{1}} \mathbb{E}_{n_{2}, n_{2}^{\prime} \in \Lambda_{2}} f\left(a+n_{1}+n_{2}\right) \overline{f\left(a+n_{1}+n_{2}^{\prime}\right) f\left(a+n_{1}^{\prime}+n_{2}\right)} f\left(a+n_{1}^{\prime}+n_{2}^{\prime}\right)$. 
Roughly speaking, the local Gowers $U^{2}$-norm counts those parallelograms with one side restricted to a narrower Bohr set $\Lambda_{1}$, and the other side restricted to an even narrower Bohr set $\Lambda_{2}$.

Clearly the local Gowers $U^{2}$-norm is always bounded by 1 . We now turn to proving an inverse theorem, which says that if the local Gowers $U^{2}$-norm of a function $f$ is large, then $f$ must possess some structure.

Theorem 3.2 (Inverse theorem for the $U^{2}$-norm, local version). Let $0<\eta<1$ be a parameter. Let $\Lambda, \Lambda_{1}=c_{1} \Lambda_{1}, \Lambda_{2}=c_{2} \Lambda_{1}$ be regular Bohr sets of dimension $d$, where $0<c_{1} \leq \eta^{8} / 5000 d$ and $0<c_{2} \leq \eta^{2} / 400 d$. If $\|f\|_{U^{2}\left(\Lambda, \Lambda_{1}, \Lambda_{2}\right)} \geq \eta$, then

$$
\mathbb{E}_{a \in \Lambda} \sup _{y \in \mathbb{R}}\left|\mathbb{E}_{n_{2} \in \Lambda_{2}} f\left(a+n_{2}\right) e\left(n_{2} y\right)\right|^{2} \geq \frac{\eta^{8}}{40}
$$

When $\Lambda, \Lambda_{1}, \Lambda_{2}$ are replaced by a finite abelian group $G$, we recover the following inverse theorem for the usual Gowers $U^{2}$-norm: if $\|f\|_{U^{2}} \geq \eta$ for a 1-bounded function $f: G \rightarrow \mathbb{C}$, then $|\hat{f}(\gamma)| \gg \eta^{4}$ for some character $\gamma \in \hat{G}$. This is slightly weaker than the usual inverse theorem which says that $|\hat{f}(\gamma)| \gg \eta^{2}$. An improvement in the exponent will lead to an improvement in the exponent in the final bound in Theorem 1.2 as well. This is, however, not our major concern.

Proof. By the definition (3.1), for at least $\eta^{4}|\Lambda| / 2$ values of $a \in \Lambda$ we have

$$
\mathbb{E}_{n_{1}, n_{1}^{\prime} \in \Lambda_{1}} \mathbb{E}_{n_{2}, n_{2}^{\prime} \in \Lambda_{2}} f\left(a+n_{1}+n_{2}\right) \overline{f\left(a+n_{1}+n_{2}^{\prime}\right) f\left(a+n_{1}^{\prime}+n_{2}\right)} f\left(a+n_{1}^{\prime}+n_{2}^{\prime}\right) \geq \frac{\eta^{4}}{2}
$$

Fix such an $a$ and we will work with condition (3.2). We may assume that $f$ is supported on the set $a+\Lambda_{1}+\Lambda_{2}$, whose size is bounded by $\left|\Lambda_{1}\right|\left(1+100 d c_{2}\right)$. Consider

$$
\sum_{n_{1}, n_{1}^{\prime} \in \mathbb{Z}} \sum_{n_{2}, n_{2}^{\prime} \in \Lambda_{2}} f\left(a+n_{1}+n_{2}\right) \overline{f\left(a+n_{1}+n_{2}^{\prime}\right) f\left(a+n_{1}^{\prime}+n_{2}\right)} f\left(a+n_{1}^{\prime}+n_{2}^{\prime}\right) .
$$

The contributions from $n_{1}, n_{1}^{\prime} \in \Lambda_{1}$ are at least $\eta^{4}\left|\Lambda_{1}\right|^{2}\left|\Lambda_{2}\right|^{2} / 2$ by (3.2). If $n_{1}, n_{1}^{\prime} \notin \Lambda_{1}$, then we must have $n_{1}, n_{1}^{\prime} \in \Lambda_{1}+2 \Lambda_{2}$ in order for the summand not to vanish. There are at most $\left(200 d c_{2}\right)^{2}\left|\Lambda_{1}\right|^{2}$ such pairs $\left(n_{1}, n_{1}^{\prime}\right)$. Hence (3.3) is at least

$$
\left[\eta^{4} / 2-\left(200 d c_{2}\right)^{2}\right]\left|\Lambda_{1}\right|^{2}\left|\Lambda_{2}\right|^{2} \geq \frac{\eta^{4}}{4}\left|\Lambda_{1}\right|^{2}\left|\Lambda_{2}\right|^{2},
$$

provided that $c_{2} \leq \eta^{2} / 400 d$.

On the other hand, we claim that (3.3) is equal to

$$
\int_{0}^{1} \int_{0}^{1}\left|\hat{\Lambda}_{2}(x)\right|^{2}|\hat{f}(y)|^{2}|\hat{f}(x+y)|^{2} d x d y
$$

Indeed, the integral above can be written as

$$
\sum_{n_{2}, n_{2}^{\prime} \in \Lambda_{2}} \sum_{r, s, t, u \in \mathbb{Z}} f(r) \overline{f(s)} f(t) \overline{f(u)} \int_{0}^{1} \int_{0}^{1} e\left(\left(n_{2}-n_{2}^{\prime}\right) x+(r-s) y+(t-u)(x+y)\right) d x d y .
$$


By orthogonality the integral above vanishes unless $u-t=n_{2}-n_{2}^{\prime}$ and $r+t=s+u$. After making the change of variables $n_{1}=r-a-n_{2}$ and $n_{1}^{\prime}=u-a-n_{2}$, it is evident that the expression above equals (3.3).

It then follows that

$$
\begin{aligned}
\frac{\eta^{4}}{4}\left|\Lambda_{1}\right|^{2}\left|\Lambda_{2}\right|^{2} & \leq \int_{0}^{1} \int_{0}^{1}\left|\hat{\Lambda}_{2}(x)\right|^{2}|\hat{f}(y)|^{2}|\hat{f}(x+y)|^{2} d x d y \\
& \leq \int_{0}^{1}|\hat{f}(y)|^{2} \cdot \sup _{y} \int_{0}^{1}\left|\hat{\Lambda}_{2}(x)\right|^{2}|\hat{f}(x+y)|^{2} d x \\
& \leq\left|\Lambda_{1}\right|\left(1+100 d c_{2}\right) \sup _{y} \int_{0}^{1}\left|\hat{\Lambda}_{2}(x)\right|^{2}|\hat{f}(x+y)|^{2} d x .
\end{aligned}
$$

Hence there exists $y=y(a) \in \mathbb{R}$ such that

$$
\int_{0}^{1}\left|\hat{\Lambda}_{2}(x)\right|^{2}|\hat{f}(x+y)|^{2} d x \geq \frac{\eta^{4}}{5}\left|\Lambda_{1}\right|\left|\Lambda_{2}\right|^{2} .
$$

If we write $f_{y}$ for the function $f_{y}(a)=f(a) e(a y)$, then the left side above is

$$
\left\|\hat{\Lambda}_{2} \cdot \hat{f}_{y}\right\|_{2}^{2}=\left\|\widehat{\Lambda_{2} * f_{y}}\right\|_{2}^{2}=\sum_{n \in \mathbb{Z}}\left|\left(\Lambda_{2} * f_{y}\right)(n)\right|^{2}=\sum_{n \in a+\Lambda_{1}+2 \Lambda_{2}}\left|\left(\Lambda_{2} * f_{y}\right)(n)\right|^{2} .
$$

Observe that

$$
\left|\left(\Lambda_{2} * f_{y}\right)(n)\right|=\left|\sum_{n_{2} \in \Lambda_{2}} f_{y}\left(n+n_{2}\right)\right|=\left|\sum_{n_{2} \in \Lambda_{2}} f\left(n+n_{2}\right) e\left(n_{2} y\right)\right| .
$$

Combining this with (3.4) and (3.5) we get

$$
\mathbb{E}_{n \in a+\Lambda_{1}+2 \Lambda_{2}}\left|\mathbb{E}_{n_{2} \in \Lambda_{2}} f\left(n+n_{2}\right) e\left(n_{2} y\right)\right|^{2} \geq \frac{\eta^{4}}{5} \cdot \frac{\left|\Lambda_{1}\right|}{\left|\Lambda_{1}+2 \Lambda_{2}\right|} \geq \frac{\eta^{4}}{10}
$$

Recall that the above is true for at least $\eta^{4}|\Lambda| / 2$ values of $a \in \Lambda$. We have thus shown that

$$
\mathbb{E}_{a \in \Lambda} \mathbb{E}_{n \in a+\Lambda_{1}+2 \Lambda_{2}} \sup _{y \in \mathbb{R}}\left|\mathbb{E}_{n_{2} \in \Lambda_{2}} f\left(n+n_{2}\right) e\left(n_{2} y\right)\right|^{2} \geq \frac{\eta^{8}}{20}
$$

After changing the order of summation over $a$ and $n$ the left side above is at most

$$
\mathbb{E}_{n \in \Lambda} \sup _{y \in \mathbb{R}}\left|\mathbb{E}_{n_{2} \in \Lambda_{2}} f\left(n+n_{2}\right) e\left(n_{2} y\right)\right|^{2}+300 d c_{1} .
$$

The proof is completed by combining this with (3.6).

Remark 3.3. In the proof above it is crucial that the variables $n_{2}, n_{2}^{\prime}$ are restricted to an even narrower Bohr set than the variables $n_{1}, n_{1}^{\prime}$. One might think that a more natural way to define the local Gowers $U^{2}$-norm is by restricting $n_{1}, n_{2}, n_{1}^{\prime}, n_{2}^{\prime}$ to the same Bohr set. An inverse theorem for this definition is obtained by Green-Tao [7], but with a significantly more involved argument. We will be content with our slightly less orthodox definition (3.1). 


\section{A Local Generalized von-Neumann Theorem}

Fix a positive integer $s \geq 2$. We will be looking for $s$-configurations $\left\{n_{i}+n_{j}+a: 1 \leq i \leq\right.$ $j \leq s\}$, where $a \in \Lambda$ and $n_{i} \in \Lambda_{i}$. Here $\Lambda, \Lambda_{1}, \cdots, \Lambda_{s}$ are all regular Bohr sets of dimension $d$, and $\Lambda_{1}=c_{1} \Lambda, \Lambda_{i}=c_{i} \Lambda_{i-1}$ for $2 \leq i \leq s$, where $0<c_{1}, \cdots, c_{s}<1 / 100 d$. To this end, define the counting function

$$
T_{s}\left(\mathcal{F} ; \Lambda, \Lambda_{1}, \cdots, \Lambda_{s}\right)=\mathbb{E}_{a \in \Lambda} \mathbb{E}_{n_{1} \in \Lambda_{1}} \cdots \mathbb{E}_{n_{s} \in \Lambda_{s}} \prod_{1 \leq i \leq j \leq s} f_{i j}\left(n_{i}+n_{j}+a\right),
$$

where $\mathcal{F}=\left\{f_{i j}\right\}$ is a collection of 1-bounded functions.

Proposition 4.1 (Generalized von-Neumann, local version). For any $1 \leq i<j \leq s$ we have $\left|T_{s}\left(\mathcal{F} ; \Lambda_{1}, \cdots, \Lambda_{s}\right)\right| \leq\left\|f_{i j}\right\|_{U^{2}\left(\Lambda, \Lambda_{i}, \Lambda_{j}\right)}$.

If one wants to control $T_{s}(\mathcal{F})$ by $f_{i i}$, it seems that a different definition of the local $U^{2}$-norm is needed (see Remark 3.3. Fortunately it is enough to have the result for $i \neq j$.

Proof. For notational convenience assume that $(i, j)=(1,2)$; the other cases are treated in the same way. We write

$$
T_{s}(\mathcal{F})=\mathbb{E}_{a \in \Lambda} \mathbb{E}_{n_{2} \in \Lambda_{2}} \cdots \mathbb{E}_{n_{s} \in \Lambda_{s}} \prod_{2 \leq i \leq j \leq s} f_{i j}\left(n_{i}+n_{j}+a\right) \mathbb{E}_{n_{1} \in \Lambda_{1}} \prod_{j=1}^{s} f_{1 j}\left(n_{1}+n_{j}+a\right) .
$$

By Cauchy-Schwarz,

$$
\left|T_{s}(\mathcal{F})\right|^{2} \leq \mathbb{E}_{a \in \Lambda} \mathbb{E}_{n_{2} \in \Lambda_{2}} \cdots \mathbb{E}_{n_{s} \in \Lambda_{s}}\left|\mathbb{E}_{n_{1} \in \Lambda_{1}} \prod_{j=1}^{s} f_{1 j}\left(n_{1}+n_{j}+a\right)\right|^{2} .
$$

Expanding the square and rearranging the order of summation we get

$$
\left|T_{s}(\mathcal{F})\right|^{2} \leq \mathbb{E}_{a \in \Lambda} \mathbb{E}_{n_{1}, n_{1}^{\prime} \in \Lambda_{1}}\left|\mathbb{E}_{n_{2} \in \Lambda_{2}} f_{12}\left(n_{1}+n_{2}+a\right) \overline{f_{12}\left(n_{1}^{\prime}+n_{2}+a\right)}\right|
$$

Apply Cauchy-Schwarz again to get

$$
\left|T_{s}(\mathcal{F})\right|^{4} \leq \mathbb{E}_{a \in \Lambda} \mathbb{E}_{n_{1}, n_{1}^{\prime} \in \Lambda^{\prime}}\left|\mathbb{E}_{n_{2} \in \Lambda^{\prime}} f_{12}\left(n_{1}+n_{2}+a\right) \overline{f_{12}\left(n_{1}^{\prime}+n_{2}+a\right)}\right|^{2}
$$

The right side above, after expanding out the square, is exactly $\|f\|_{U^{2}\left(\Lambda, \Lambda_{1}, \Lambda_{2}\right)}^{4}$.

For a Bohr set $\Lambda$, we write $2 \cdot \Lambda$ for the set $\{2 n: n \in \Lambda\}$. Note that this is contained in, but usually much smaller than the dilated set $2 \Lambda$.

Corollary 4.2 (Dichotomy, local version). Let $s \geq 2$ be a positive integer. Let $\Lambda$ be a regular Bohr set of dimension d and $A \subset \Lambda$ be a subset with $|A|=\delta|\Lambda|$. Let $0<c_{1}, \cdots, c_{s}<1 / 100 d$ be real parameters with $c_{1} \leq \delta^{s} / 3200 d s^{2}$. Set $\Lambda_{1}=c_{1} \Lambda, \Lambda_{i}=c_{i} \Lambda_{i-1}$ for $2 \leq i \leq s$. Suppose that $A$ does not contain any nontrivial s-configurations. Then either one of the following statements holds:

(1) $\left|\Lambda_{s}\right| \leq 32 s^{2} \delta^{-\left(\begin{array}{c}s+1 \\ 2\end{array}\right)}$; 
(2) The density of $A$ on $a+2 \cdot \Lambda_{i}$ is at least $\left(1+1 / 8 s^{2}\right) \delta$ for some $a \in \Lambda$ and $1 \leq i \leq s$ with $a+2 \cdot \Lambda_{i} \subset \Lambda$;

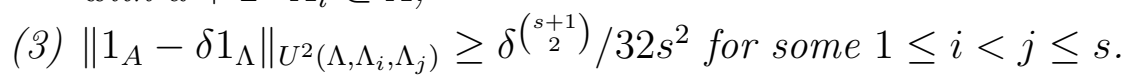

Proof. Assume that case (1) fails. Since $A$ does not contain any non-trivial $d$-configurations, we have

$$
T_{s}\left(1_{A}\right) \leq \frac{s^{2}}{\left|\Lambda_{s}\right|} \leq \frac{1}{32} \delta^{\left(\begin{array}{c}
s+1 \\
2
\end{array}\right)}
$$

On the other hand, we have the decomposition into $L+1=\left(\begin{array}{l}s \\ 2\end{array}\right)+1$ components

$$
T_{s}\left(1_{A}\right)=T_{s}\left(\mathcal{F}_{1}\right)+\cdots+T_{s}\left(\mathcal{F}_{L}\right)+T_{s}(\mathcal{G}),
$$

such that each $\mathcal{F}_{k}$ has $1_{A}-\delta 1_{\Lambda}$ as the $i j$-component function for some $i<j$, and $\mathcal{G}=\left(g_{i j}\right)$ satisfies $g_{i j}=\delta 1_{\Lambda}$ for all $i<j$ and $g_{i i}=1_{A}$. Using Proposition 4.1 we get

$$
T_{s}\left(1_{A}\right) \geq T_{s}(\mathcal{G})-s^{2}\left\|1_{A}-\delta 1_{\Lambda}\right\|_{U^{2}\left(\Lambda, \Lambda_{i}, \Lambda_{j}\right)}
$$

for some $1 \leq i<j \leq s$. To estimate $T_{s}(\mathcal{G})$, note that if $a \in\left(1-2 c_{1}\right) \Lambda$ and $n_{i}, n_{j} \in \Lambda_{1}$, then $a+n_{i}+n_{j} \in \Lambda$. Hence by the regularity of $\Lambda$,

$$
T_{s}(\mathcal{G}) \geq \delta^{\left(\begin{array}{c}
s \\
2
\end{array}\right)}\left[\mathbb{E}_{a \in \Lambda} \prod_{i=1}^{s}\left(\mathbb{E}_{n \in \Lambda_{i}} 1_{A}\left(2 n_{i}+a\right)\right)-200 d c_{1}\right]
$$

Now write $\delta_{i}(a)$ for the density of $A$ on $a+2 \cdot \Lambda_{i}$, and let $E_{i}$ be the set of $a \in\left(1-2 c_{1}\right) \Lambda$ with $\delta_{i}(a)<(1-1 / s) \delta$. Assume that case $(2)$ of the conclusion fails, so that $\delta_{i}(a) \leq\left(1+1 / 8 s^{2}\right) \delta$ for all $a \in\left(1-2 c_{1}\right) \Lambda$. Since

$$
\mathbb{E}_{a \in \Lambda} \delta_{i}(a)=\mathbb{E}_{n \in \Lambda_{i}} \mathbb{E}_{a \in \Lambda} 1_{A}(2 n+a) \geq \delta-200 d c_{1} \geq\left(1-1 / 4 s^{2}\right) \delta,
$$

we have

$$
\left(1-1 / 4 s^{2}\right) \delta \leq \frac{\left|E_{i}\right|}{|\Lambda|}(1-1 / s) \delta+\left(1-\frac{\left|E_{i}\right|}{|\Lambda|}\right)\left(1+1 / 8 s^{2}\right) \delta+200 d c_{1}
$$

From this it follows that $\left|E_{i}\right| \leq|\Lambda| / 2 s$. Let $E$ be the union $E_{1} \cup \cdots E_{s}$. Then $|E| \leq|\Lambda| / 2$. Hence

$$
T_{s}(\mathcal{G}) \geq \delta^{\left(\begin{array}{c}
s \\
2
\end{array}\right)}\left(\frac{1}{2} \mathbb{E}_{a \in \Lambda \backslash E} \delta_{1}(a) \cdots \delta_{d}(a)-200 d c_{1}\right) \geq \delta^{\left(\begin{array}{c}
s \\
2
\end{array}\right)}\left(\frac{1}{8} \delta^{s}-200 d c_{1}\right) \geq \frac{1}{16} \delta^{\left(\begin{array}{c}
s+1 \\
2
\end{array}\right)} .
$$

Combining this with (4.1) and (4.2) we get case (iii) of the conclusion.

Compared with the usual dichotomy in Roth's argument, the second case above is new. However it is certainly harmless as it already implies a density increment. 


\section{Obtaining Density Increment in the Local Setting}

The iterative procedure of attacking Theorem 1.2 is the following. Suppose that $A$ does not contain a nontrivial $s$-configuration. Then Corollary 4.2 implies that there is a large local Gowers $U^{2}$-norm. By Theorem 3.2 we thus have a large Fourier coefficient (in the average sense). Finally, this large Fourier coefficient leads to a density increment on a smaller Bohr set. This final step of the argument is recorded in the following lemma.

Lemma 5.1 (Large Fourier coefficient leads to density increment, local version). Let $0<$ $\eta<1$ be a parameter. Let $\Lambda=\Lambda_{\theta, \epsilon, M}, \Lambda_{1}=c_{1} \Lambda$ be regular Bohr sets of dimension $d$, where $c_{1} \leq 2^{-15} \eta^{3} d^{-1}$. Suppose that $\mathbb{E}_{a \in \Lambda} f(a)=0$. If

$$
\mathbb{E}_{a \in \Lambda} \sup _{y \in \mathbb{R}}\left|\mathbb{E}_{n_{1} \in \Lambda_{1}} f\left(a+n_{1}\right) e\left(n_{1} y\right)\right|^{2} \geq \eta^{2},
$$

then either one of the following statements holds:

(1) $f$ has density increment on a translate of $\Lambda_{1}$ : $\mathbb{E}_{n_{1} \in \Lambda_{1}} f\left(a+n_{1}\right) \geq \eta^{3} / 128$ for some a with $a+\Lambda_{1} \subset \Lambda$;

(2) for any positive $c^{\prime} \leq 2^{-13} \eta d^{-1}$, $f$ has density increment on some translate of a $(d+1)$ dimensional Bohr set $\Lambda^{\prime}=\Lambda_{\theta^{\prime}, \epsilon^{\prime}, M^{\prime}}$ with $\epsilon^{\prime}=c^{\prime} c_{1} \epsilon$ and $M^{\prime}=c^{\prime} c_{1} M$ :

$$
\mathbb{E}_{n^{\prime} \in \Lambda^{\prime}} f\left(a+n^{\prime}\right) \geq \eta / 16
$$

for some a with $a+\Lambda^{\prime} \subset \Lambda$.

Proof. For $a \in \Lambda$ write $\delta(a)=\mathbb{E}_{n_{1} \in \Lambda_{1}} f\left(a+n_{1}\right)$. Suppose that case (i) fails, so that $\delta(a) \leq$ $\eta^{3} / 128$ for each $a \in\left(1-c_{1}\right) \Lambda$. Let $E$ be the set of $a \in\left(1-c_{1}\right) \Lambda$ with $\delta(a) \leq-\eta / 32$. Note that

$$
\mathbb{E}_{a \in \Lambda} \delta(a)=\mathbb{E}_{a \in \Lambda} \mathbb{E}_{n_{1} \in \Lambda_{1}} f\left(a+n_{1}\right) \geq-200 c_{1} d \geq-\eta^{3} / 128
$$

since $\mathbb{E}_{a \in \Lambda} f(a)=0$. This gives the upper bound $|E| \leq 3 \eta^{2}|\Lambda| / 4$. It follows that there exists $a \in\left(1-c_{1}\right) \Lambda \backslash E$ and $y \in \mathbb{R}$ such that

$$
\left|\mathbb{E}_{n_{1} \in \Lambda_{1}} f\left(a+n_{1}\right) e\left(n_{1} y\right)\right| \geq \eta / 2 .
$$

Fix such an $a$ and $y$. Define $\Lambda^{\prime}$ by taking $\theta^{\prime}=(\theta, y) \in \mathbb{R}^{d+1}$. Then for any $n^{\prime} \in \Lambda^{\prime}$,

$$
\left|\mathbb{E}_{n_{1} \in \Lambda_{1}} f\left(a+n_{1}+n^{\prime}\right) e\left(\left(n_{1}+n^{\prime}\right) y\right)-\mathbb{E}_{n_{1} \in \Lambda_{1}} f\left(a+n_{1}\right) e\left(n_{1} y\right)\right| \leq 200 c^{\prime} d .
$$

After averaging over $n^{\prime}$ and changing the order of summation we get

$$
\left|\mathbb{E}_{n_{1} \in \Lambda_{1}} \mathbb{E}_{n^{\prime} \in \Lambda^{\prime}} f\left(a+n_{1}+n^{\prime}\right) e\left(\left(a+n_{1}+n^{\prime}\right) y\right)\right| \geq \mathbb{E}_{n_{1} \in \Lambda_{1}} f\left(a+n_{1}\right) e\left(n_{1} y\right)-200 c^{\prime} d \geq \frac{\eta}{4} .
$$

On the other hand, for $n^{\prime} \in \Lambda^{\prime}$ we have $\left|1-e\left(y n^{\prime}\right)\right| \leq 8 \epsilon^{\prime}$. Hence

$$
\begin{aligned}
\left|\mathbb{E}_{n_{1} \in \Lambda_{1}} \mathbb{E}_{n^{\prime} \in \Lambda^{\prime}} f\left(a+n_{1}+n^{\prime}\right) e\left(\left(a+n_{1}+n^{\prime}\right) y\right)\right| & \leq \mathbb{E}_{n_{1} \in \Lambda_{1}}\left|\mathbb{E}_{n^{\prime} \in \Lambda^{\prime}} f\left(a+n_{1}+n^{\prime}\right) e\left(n^{\prime} y\right)\right| \\
& \leq \mathbb{E}_{n_{1} \in \Lambda_{1}}\left|\mathbb{E}_{n^{\prime} \in \Lambda^{\prime}} f\left(a+n_{1}+n^{\prime}\right)\right|+8 \epsilon^{\prime}
\end{aligned}
$$

It then follows that

$$
\mathbb{E}_{n_{1} \in \Lambda_{1}}\left|\mathbb{E}_{n^{\prime} \in \Lambda^{\prime}} f\left(a+n_{1}+n^{\prime}\right)\right| \geq \frac{\eta}{8}
$$


Note that

$$
\mathbb{E}_{n_{1} \in \Lambda_{1}} \mathbb{E}_{n^{\prime} \in \Lambda^{\prime}} f\left(a+n_{1}+n^{\prime}\right) \geq \delta(a)-200 c^{\prime} d \geq-\frac{\eta}{32}-200 c^{\prime} d \geq-\frac{\eta}{16}
$$

since $a \notin E$. Hence there exists $n_{1} \in \Lambda_{1}$ such that

$$
\mathbb{E}_{n^{\prime} \in \Lambda^{\prime}} f\left(a+n_{1}+n^{\prime}\right) \geq \frac{\eta}{16} \text {. }
$$

Proposition 5.2 (Iterative step for Theorem 1.2). Let $\Lambda=\Lambda_{\theta, \epsilon, M}$ be a regular Bohr set of dimension $d$ and let $A \subset \Lambda$ be a subset with $|A|=\delta|\Lambda|$. Set

$$
x_{1}=2^{-85} s^{-24} d^{-1} \delta^{6 s(s+1)}, \quad x_{2}=\cdots=x_{s}=2^{-20} s^{-4} d^{-1} \delta^{s(s+1)} .
$$

Then there exists $c_{i} \in\left[x_{i}, 2 x_{i}\right]$ such that the Bohr sets $\Lambda_{1}=c_{1} \Lambda$ and $\Lambda_{i}=c_{i} \Lambda_{i-1}(2 \leq i \leq s)$ are all regular. Moreover, either one of the following statements holds:

(1) A contains a non-trivial s-configuration;

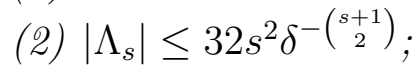

(3) the density of $A$ on $a+2 \cdot \Lambda_{i}$ is at least $\left(1+1 / 8 s^{2}\right) \delta$ for some $a \in \Lambda$ and $1 \leq i \leq s$;

(4) the density of $A$ on $a+\Lambda_{i}$ is at least $\delta+2^{-54} s^{-16} \delta^{4 s(s+1)}$ for some $a \in \Lambda$ and $1 \leq i \leq s$;

(5) there is a regular Bohr set $\Lambda^{\prime}=\Lambda_{\theta^{\prime}, \epsilon^{\prime}, M^{\prime}}$ of dimension $d+1$, where $\epsilon^{\prime} \geq c^{\prime} c_{1} \cdots c_{s} \epsilon$, $M^{\prime} \geq c^{\prime} c_{1} \cdots c_{s} M$ with

$$
c^{\prime} \geq 2^{-37} s^{-8} d^{-1} \delta^{2 s(s+1)}
$$

such that the density of $A$ on $a+\Lambda^{\prime}$ is at least $\delta+2^{-28} s^{-8} \delta^{2 s(s+1)}$ for some a.

Proof. Assume that case (1) fails. By Lemma 2.4 we may choose $c_{i} \in\left[x_{i}, 2 x_{i}\right](1 \leq i \leq s)$ such that $\Lambda_{1}=c_{1} \Lambda, \Lambda_{2}=c_{2} \Lambda_{1}, \cdots$ are all regular Bohr sets. By Corollary 4.2 , either we are in case $(2)$ or $(3)$, or

$$
\left\|1_{A}-\delta 1_{\Lambda}\right\|_{U^{2}\left(\Lambda, \Lambda_{i}, \Lambda_{j}\right)} \geq \delta^{\left(\begin{array}{c}
s+1 \\
2
\end{array}\right)} / 32 s^{2}
$$

for some $1 \leq i<j \leq s$. By Theorem 3.2 ,

$$
\mathbb{E}_{a \in \Lambda} \sup _{y \in \mathbb{R}}\left|\mathbb{E}_{n_{j} \in \Lambda_{j}} f\left(a+n_{j}\right) e\left(n_{j} y\right)\right|^{2} \geq 2^{-46} s^{-16} \delta^{4 s(s+1)} .
$$

Finally apply Proposition 5.1 to arrive at case (4) or (5).

Proof of Theorem 1.2. Set

$$
d_{0}=1, \quad \epsilon_{0}=1, \quad M_{0}=N, \quad \delta_{0}=\delta / 2 .
$$

Start with the $d_{0}$-dimensional regular Bohr set $\Lambda_{0}=\Lambda_{1, \epsilon_{0}, M_{0}}=[-N, N]$. Let $A_{0}=A$ so that the density of $A_{0}$ in $\Lambda_{0}$ is $\delta_{0}$. We will apply Proposition 5.2 repeatedly to obtain sequences of Bohr sets $\Lambda_{0}, \Lambda_{1} \cdots, \Lambda_{k}$ and subsets $A_{1} \subset \Lambda_{1}, \cdots, A_{k} \subset \Lambda_{k}$ of densities $\delta_{1}, \cdots, \delta_{k}$ satisfying the following properties:

(1) (dimension bound) $d_{i} \leq d_{i-1}+1$ for each $i=1,2, \cdots, k$; 
(2) (density increment) for each $i=1,2, \cdots, k$ we have

(3) (size bound) $\epsilon_{i} \geq c \epsilon_{i-1}$ and $M_{i} \geq c M_{i-1}$ for each $i=1,2, \cdots, k$, where

$$
\delta_{i} \geq \delta_{i-1}+2^{-54} s^{-16} \delta_{i-1}^{4 s(s+1)}
$$

moreover if $d_{i}=d_{i-1}+1$ then

$$
\delta_{i} \geq \delta_{i-1}+2^{-28} s^{-8} \delta_{i-1}^{2 s(s+1)}
$$

$c=s^{-100 s} d_{k}^{-s} \delta^{10 s^{3}}$

(4) for each $i=1,2, \cdots, k$, existence of nontrivial $s$-configurations in $A_{i}$ implies existence of nontrivial $s$-configurations in $A_{i-1}$;

(5) (end of iterations) either $A_{k}$ contains a nontrivial $s$-configuration, or

$$
\left|c \Lambda_{k}\right| \leq 32 s^{2} \delta^{-\left(\begin{array}{c}
s+1 \\
2
\end{array}\right)},
$$

where the constant $c$ is defined in (5.3).

It remains to show that (5.4) does not occur. This will imply that $A_{k}$ contains a nontrivial $s$-configuration, and thus so does the original set $A$. By (5.1), (5.2), and (5.3), we have

$$
\begin{gathered}
k \leq 2^{55} s^{16} \delta^{-4 s(s+1)}, \\
d_{k} \leq 2^{29} s^{8} \delta^{-2 s(s+1)}, \\
\epsilon_{k} \geq c^{k}, \quad M_{k} \geq c^{k} N .
\end{gathered}
$$

By Lemma 2.2 we have the lower bound

$$
\left|c \Lambda_{k}\right| \geq\left(c \epsilon_{k}\right)^{d_{k}} M_{k} \geq c^{(k+1) d_{k}} c^{k} N \geq N \exp \left(-2^{94} s^{27} \delta^{-6 s(s+1)} \log (1 / \delta)\right) .
$$

The inequality

$$
N \exp \left(-2^{94} s^{27} \delta^{-6 s(s+1)} \log (1 / \delta)\right)>32 s^{2} \delta^{-\left(\begin{array}{c}
s+1 \\
2
\end{array}\right)}
$$

can be readily verified under the assumption on $\delta$. This shows that (5.4) cannot happen.

\section{Proof of Corollary 1.3}

The general strategy of proving Corollary 1.3 is the same as in [16] (see also [3]). It follows from the following proposition, just as Theorem 1.1 in [16] follows from Theorem 1.2 there.

Proposition 6.1. Let h be a sufficiently large positive integer. Let $X, Y$ be two finite subsets of positive integers with

$$
h^{-29}|Y| \geq|X| \geq \exp \left(\exp \left(C h^{2} \log h\right)\right)
$$

for some sufficiently large constant $C>0$. Then $Y$ contains a subset $Z$ of size $|Z|=h$ disjoint from $X$ and is sumfree with respect to the union $X \cup Y$. In other words, $z_{1}+z_{2} \notin X \cup Y$ for distinct $z_{1}, z_{2} \in Z$. 
Using the notation in [16], we could take $F(h)=\exp \left(\exp \left(C h^{2} \log h\right)\right)$, and thus Corollary 1.3 is true with the lower bound $|B| \geq g(n) \log n$, where $g(n)=\mathrm{cm} / \log m$. Here $c$ is a small constant and $F(m)=n^{1 / 2}$. Thus $m$ can be taken to be $(\log \log n)^{1 / 2-o(1)}$. We now focus on proving Proposition 6.1.

Proposition 6.2. Let $h, X, Y$ be as in the statement of Theorem 6.1. Suppose that $Y$ does not contain any subset $Z$ of size $h$ which is sumfree with respect to $X \cup Y$. Then there is a subset $Y_{3} \subset Y$ satisfying the following properties:

(1) (large size) $\left|Y_{3}\right| \gg h^{-29}|Y|$;

(2) (small doubling) $\left|Y_{3}+Y_{3}\right| \ll h^{181}\left|Y_{3}\right|$;

(3) the set $2 \cdot Y_{3}$ is disjoint from $X \cup Y$. In other words, $2 y_{3} \notin X \cup Y$ for any $y_{3} \in Y_{3}$.

Proof. See Section 6 in [16].

To deduce Proposition 6.1 from Proposition 6.2, it suffices to find an $h$-configuration in $Y_{3}$. The small doubling property of $Y_{3}$ allows us to extract a subset of $Y_{3}$ lying densely inside an interval. This is achieved in [16] by an application of Freiman's theorem. A more economical way of doing this is to use an embedding lemma due to Ruzsa [12,13] (see also Lemma 5.26 in $[19]$ ).

Lemma 6.3 (Ruzsa's embedding lemma). Let $A \subset \mathbb{Z}$ be a finite set with $|A-A| \leq K|A|$. Then there is a subset $A^{\prime} \subset A$ with $\left|A^{\prime}\right| \geq|A| / 2$ such that $A^{\prime}$ is Freiman isomorphic to a subset $A^{\prime \prime} \subset \mathbb{Z} / N \mathbb{Z}$ with $N \ll K|A|$. In other words, there is a bijection $\phi: A^{\prime} \rightarrow A^{\prime \prime}$ such that

$$
a_{1}^{\prime}+a_{2}^{\prime}=a_{3}^{\prime}+a_{4}^{\prime} \Longleftrightarrow \phi\left(a_{1}^{\prime}\right)+\phi\left(a_{2}^{\prime}\right)=\phi\left(a_{3}^{\prime}\right)+\phi\left(a_{4}^{\prime}\right)
$$

whenever $a_{1}^{\prime}, a_{2}^{\prime}, a_{3}^{\prime}, a_{4}^{\prime} \in A^{\prime}$.

Proof of Proposition 6.1. As noted above, it suffices to show that $Y_{3}$ contains a nontrivial $h$ configuration. Since $\left|Y_{3}+Y_{3}\right| \ll h^{181}\left|Y_{3}\right|$, we have $\left|Y_{3}-Y_{3}\right| \ll h^{362}\left|Y_{3}\right|$ (this is a consequence of the Ruzsa triangle inequality; see Section 2.3 in [19]). By Ruzsa's embedding lemma, there is a subset $Y_{4} \subset Y_{3}$ with $\left|Y_{4}\right| \geq\left|Y_{3}\right| / 2$ that is Freiman isomorphic to a subset $Y_{4}^{\prime} \subset \mathbb{Z} / N \mathbb{Z}$ with $N \ll h^{362}\left|Y_{3}\right|$.

Identify $\mathbb{Z} / N \mathbb{Z}$ with $[N]$ and view $Y_{4}^{\prime}$ as a subset of the integers. To find a nontrivial $h$-configuration in $Y_{3}$, it suffices to find a nontrivial $h$-configuration in $Y_{4}^{\prime}$. The density of $Y_{4}^{\prime}$ in $[N]$ is

$$
\delta=\frac{\left|Y_{4}^{\prime}\right|}{N} \gg h^{-362}
$$

By Theorem 1.2, it thus suffices to show that

$$
h^{-362} \gg(\log N)^{-1 / 20 h^{2}}
$$

Simple algebra reveals that this is implied by the condition (6.1). 


\section{REFERENCES}

[1] J. Bourgain. On triples in arithmetic progression. Geom. Funct. Anal., 9(5):968-984, 1999.

[2] J. Bourgain. Roth's theorem on progressions revisited. J. Anal. Math., 104:155-192, 2008.

[3] J. Dousse. On a generalisation of Roth's theorem for arithmetic progressions and applications to sum-free subsets. Math. Proc. Camb. Phil. Soc., 2013. To appear.

[4] W. T. Gowers. A new proof of Szemerédi's theorem for arithmetic progressions of length four. Geom. Funct. Anal., 8(3):529-551, 1998.

[5] W. T. Gowers. A new proof of Szemerédi's theorem. Geom. Funct. Anal., 11(3):465-588, 2001.

[6] B. Green and T. Sanders. A quantitative version of the idempotent theorem in harmonic analysis. Ann. of Math. (2), 168(3):1025-1054, 2008.

[7] B. Green and T. Tao. Personal communication.

[8] B. Green and T. Tao. New bounds for Szemerédi's theorem. II. A new bound for $r_{4}(N)$. In Analytic number theory, pages 180-204. Cambridge Univ. Press, Cambridge, 2009.

[9] B. Green and T. Tao. Linear equations in primes. Ann. of Math. (2), 171(3):1753-1850, 2010.

[10] D. R. Heath-Brown. Integer sets containing no arithmetic progressions. J. London Math. Soc. (2), 35(3):385-394, 1987.

[11] K. F. Roth. On certain sets of integers. J. London Math. Soc., 28:104-109, 1953.

[12] I. Z. Ruzsa. Arithmetic progressions in sumsets. Acta Arith., 60(2):191-202, 1991.

[13] I. Z. Ruzsa. Generalized arithmetical progressions and sumsets. Acta Math. Hungar., 65(4):379-388, 1994.

[14] T. Sanders. Three-term arithmetic progressions and sumsets. Proc. Edinb. Math. Soc. (2), 52(1):211233, 2009.

[15] T. Sanders. On Roth's theorem on progressions. Ann. of Math. (2), 174(1):619-636, 2011.

[16] B. Sudakov, E. Szemerédi, and V. H. Vu. On a question of Erdős and Moser. Duke Math. J., 129(1):129155,2005

[17] E. Szemerédi. Integer sets containing no arithmetic progressions. Acta Math. Hungar., 56(1-2):155-158, 1990.

[18] T. Tao. Higher order Fourier analysis, volume 142 of Graduate Studies in Mathematics. American Mathematical Society, Providence, RI, 2012.

[19] T. Tao and V. Vu. Additive combinatorics, volume 105 of Cambridge Studies in Advanced Mathematics. Cambridge University Press, Cambridge, 2006.

Department of Mathematics, Stanford University, 450 Serra Mall, Bldg. 380, Stanford, CA 94305-2125 